\title{
User's Guide for Hysteretic Capillary Pressure and Relative Permeability Functions in iTOUGH2
}

\author{
Christine Doughty \\ Earth Sciences Division \\ Lawrence Berkeley National Laboratory
}

August 2009 


\section{Background}

The precursor of TOUGH2, TOUGH, was originally developed with non-hysteretic characteristic curves. Hysteretic capillary pressure functions were implemented in TOUGH in the late 1980s by Niemi and Bodvarsson (1988), and hysteretic capillary pressure and relative permeability functions were added to iTOUGH2 about ten years later by Finsterle et al. (1998). Recently, modifications were made to the iTOUGH2 hysteretic formulation to make it more robust and efficient (Doughty, 2007). Code development is still underway, with the ultimate goal being a hysteretic module that fits into the standard TOUGH2 (Pruess et al., 1991) framework.

This document provides a user's guide for the most recent version of the hysteretic code, which runs within iTOUGH2 (Finsterle, 1999a,b,c). The current code differs only slightly from what was presented in Doughty (2007), hence that document provides the basic information on the processes being modeled and how they are conceptualized. This document focuses on a description of the user-specified parameters required to run hysteretic iTOUGH2. In the few instances where the conceptualization differs from that of Doughty (2007), the features described here are the current ones.

Sample problems presented in this user's guide use the equation-of-state module ECO2N (Pruess, 2005). The components present in $\mathrm{ECO} 2 \mathrm{~N}$ are $\mathrm{H}_{2} \mathrm{O}, \mathrm{NaCl}$, and $\mathrm{CO}_{2}$. Two fluid phases and one solid phase are considered: an aqueous phase, which primarily consists of liquid $\mathrm{H}_{2} \mathrm{O}$ and may contain dissolved $\mathrm{NaCl}$ and $\mathrm{CO}_{2}$; a supercritical phase which primarily consists of $\mathrm{CO}_{2}$, but also includes a small amount of gaseous $\mathrm{H}_{2} \mathrm{O}$; and a solid phase consisting of precipitated $\mathrm{NaCl}$. Details of the $\mathrm{ECO} 2 \mathrm{~N}$ formulation may be 
found in Pruess (2005). The aqueous phase is the wetting phase and is denoted 'liquid', whereas the supercritical phase is the non-wetting phase and is denoted 'gas'. The hysteretic formalism may be applied to other iTOUGH2 equation-of-state modules, as long as the liquid phase is the wetting phase and the gas phase is the non-wetting phase.

\section{Characteristic-Curve Functional Forms}

The basic equation for capillary pressure $P_{c}$ as a function of liquid saturation $S_{l}$ is adapted from the van Genuchten (1980) formulation, and is given by

$P_{c}=-P_{0}^{p}\left[\left(\frac{S_{l}-S_{l \min }}{1-S_{g r}^{\Delta}-S_{l \min }}\right)^{-\left(\frac{1}{m^{p}}\right)}-1\right]^{\left(1-m^{p}\right)}$,

where $p$ identifies the flow process, drainage ( $d$ ) or imbibition ( $w$ ), that is occurring. The parameters $P_{0}^{d}, P_{0}{ }^{w}, m^{d}, m^{w}$, and $S_{l \text { min }}$ are specified in the ROCKS or RPCAP blocks of the TOUGH2 input file (see below for details). The parameter $S_{g r}{ }^{\Delta}$, the residual gas saturation, depends on the saturation history of the grid block through the turning- point saturation $S_{l}^{\Delta}$, which identifies the transition from drainage to imbibition for a particular grid block. Following Land (1968), we take

$$
S_{g r}^{\Delta}=\frac{1}{1 /\left(1-S_{l}^{\Delta}\right)+1 / S_{g r \max }-1 /\left(1-S_{l r}\right)},
$$

where $S_{g r \max }$ and $S_{l r}$ are maximum residual gas saturation and residual liquid saturation, respectively, which are material properties specified in the ROCKS or RPCAP blocks (see below for details). Equation (2) indicates that smaller values of $S_{l}^{\Delta}$ produce larger 
values of $S_{g r}{ }^{\Delta}$. In other words, the locations that once held the most $\mathrm{CO}_{2}\left(\operatorname{small} S_{l}{ }^{\Delta}\right)$, become the locations that trap the most $\mathrm{CO}_{2}\left(\operatorname{large} S_{g r}{ }^{\Delta}\right.$ ). Equation (1) provides the socalled primary drainage and primary imbibition capillary pressure curves. During a simulation, the actual capillary pressure curve being followed is determined by interpolation between the two primary curves, as described in Finsterle et al. (1998) and illustrated in Doughty (2007). Interpolated curves are known as scanning curves. In total, four branches of the capillary pressure curve are defined: 1 - primary drainage, 2 - firstorder scanning imbibition, 3 - second-order scanning drainage, 4 - third-order scanning imbibition.

The relative permeability functions also include hysteretic effects arising from the trapped component of the gas phase that develops during imbibition. These functions are taken from Parker and Lenhard (1987) and Lenhard and Parker (1987), who adapted them from the non-hysteretic expressions of van Genuchten (1980). As implemented here, the relative permeability to liquid $k_{r l}$ is given by

$k_{r l}=\sqrt{\bar{S}}\left[1-\left(1-\frac{\bar{S}_{g t}}{1-\bar{S}_{l}^{\Lambda}}\right)\left(1-\left(\bar{S}_{l}+\bar{S}_{g t}\right)^{1 / m}\right)^{m}-\left(\frac{\bar{S}_{g t}}{1-\bar{S}_{l}^{\Lambda}}\right)\left(1-\left(\bar{S}_{l}^{\Delta}\right)^{1 / m}\right)^{m}\right]^{2}$

and the relative permeability to gas $k_{r g}$ is given by

$k_{r g}=k_{r g \max }\left(1-\left(\bar{S}_{l}+\bar{S}_{g t}\right)\right)^{\gamma}\left(1-\left(\bar{S}_{l}+\bar{S}_{g t}\right)^{1 / m}\right)^{2 m}$,

where $\bar{S}_{l}$ and $\bar{S}_{l}^{\Delta}$ are effective values of liquid saturation $S_{l}$ and turning-point saturation $S_{l}^{\Delta}$, respectively, normalized with respect to residual liquid-phase saturation $S_{l r}$ : 
$\bar{S}_{l}=\frac{S_{l}-S_{l r}}{1-S_{l r}}$

$\bar{S}_{l}^{\Delta}=\frac{S_{l}^{\Delta}-S_{l r}}{1-S_{l r}}$

The parameter $\bar{S}_{g t}$ is the effective value of the trapped gas-phase saturation:

$\bar{S}_{g t}=\frac{S_{g r}^{\Delta}\left(S_{l}-S_{l}^{\Delta}\right)}{\left(1-S_{l r}\right)\left(1-S_{l}^{\Delta}-S_{g r}^{\Delta}\right)}$.

The parameters $S_{l r}, \gamma, m$, and $k_{r g m a x}$ are specified in the ROCKS or RPCAP blocks of the TOUGH2 input file (see below for details). Unlike the capillary pressure algorithm, where Equation (1) merely provides the envelope functions within which interpolation determines the actual capillary pressure curve, Equations (3) and (4) provide the actual relative permeabilities for both drainage and imbibition. The only difference between the two processes is that $\bar{S}_{g t}$ is zero for the primary drainage curve, and non-zero for the scanning curves. Moreover, $\bar{S}_{g t}$ has the same value for all scanning curves, implying that the relative permeability curves have only two branches: one for primary drainage and the other for everything else.

Equation (1) is not defined for $S_{l}<S_{l \min }$ or $S_{l}>\left(1-S_{g r}{ }^{\Delta}\right)$, Equations (3) and (4) are not defined for $S_{l}<S_{l r}$, and Equation (3) has an infinite derivative for $S_{l}=\left(1-S_{g r}{ }^{\Delta}\right)$. However, characteristic curves must be defined over the entire liquid saturation domain from zero to one, because even when a phase is immobile, and hence unable to move by pressure forces, its saturation can change due to other processes (e.g., evaporation of 
liquid water, dissolution of supercritical $\mathrm{CO}_{2}$ ). Hence, the basic hysteretic characteristic curves given by Equations (1), (3), and (4) must be augmented by extensions. Figures 1 and 2 illustrate the key features of the extended hysteretic characteristic curves.

Figure 1 shows the features of the extended hysteretic capillary pressure function. The imbibition branch $P_{c}{ }^{w}$ is illustrated for a value of $S_{l}^{\Delta}=0.3$, meaning that for this particular grid block located near a $\mathrm{CO}_{2}$ injection location, liquid saturation began at $S_{l}=$ 1, decreased until $S_{l}=0.3$ as $\mathrm{CO}_{2}$ entered the grid block, then began to increase again after $\mathrm{CO}_{2}$ injection ceased. In practice, $S_{l}^{\Delta}$ can range from $S_{l r}$ to 1 .

In Equation (1), $P_{c}$ goes to infinity at $S_{l}=S_{l \min }$. In the extended formulation, the $P_{c}$ defined by Equation (1) is smoothly connected to an exponential or power-law extension that remains finite for all values of $S_{l}$. The transition to the extension is controlled by the user-specified variable $P_{c \max }$, which is the value of Equation (1) at $S_{m}$, the point where the two functions meet. For the exponential extension, as $P_{c \max }$ increases, $S_{m}$ decreases (i.e., gets closer to $S_{l \mathrm{~min}}$ ) and the slope of Equation (1) at $S_{m}$ increases. Thus, the slope of the extension at $S_{m}$ must also increase, hence its maximum value $P_{c}\left(S_{l}=0\right)$ also increases. For the power-law extension, the user specifies the value of $P_{c}\left(S_{l}=0\right)$ explicitly. The power-law extension has zero slope at $S_{l}=0$.

At the other end of the saturation range, two user options are available for extending the imbibition branch $P_{c}{ }^{w}$ for $S_{l}>S_{l}{ }^{*}$, where $S_{l}{ }^{*}$ is defined as $1-S_{g r}{ }^{\Delta}$. Either $P_{c}{ }^{w}$ can go to zero at $S_{l}^{*}$, or $P_{c}{ }^{w}$ can gradually decline to zero at $S_{l}=1$ following a power-law function.

Figure 2 shows the features of the extended hysteretic relative permeability $\left(k_{r l}\right.$ and $k_{r g}$ ) functions. Previously (Doughty, 2007), when $S_{l}=S_{l r}, k_{r l}=0$ and $k_{r g}=1$. The 
requirement $k_{r g}=1$ at $S_{l}=S_{l r}$ has been relaxed, and the user now specifies the value of $k_{r g}\left(S_{l r}\right)$, which is denoted $k_{r g \max }$. If $k_{r g \max }=1$, there is no difference from the previous formulation. However, if $k_{r g \max }<1$, then there are two options for $k_{r g}$ for the saturation range $0<S_{l}<S_{l r}$. In either case, $k_{r g}$ increases from $k_{r g m a x}$ to 1 as $S_{l}$ decreases from $S_{l r}$ to 0 , but the variation can either be linear or cubic. If the linear option is chosen, a small cubic spline is added at $S_{l r}$, to guarantee the continuity and smoothness of the extension and original function (this spline is present in Figure 2, but is too small to see). Although $S_{l}^{\Delta}$ can be as small as $S_{l r}$, meaning that an imbibition curve could originate at $S_{l r}$, the $k_{r g}$ extension below $S_{l r}$ is only smoothly connected to the drainage branch $k_{r g}{ }^{\mathrm{d}}$. In other words, there is no hysteresis along the extension.

At the other end of the saturation range, Equation (3) cannot be used during imbibition all the way to $S_{l}=S_{l}^{*}$ because the slope $k_{r l}{ }^{w}$ becomes infinite there, which yields the non-physical consequence of ${k_{r l}}^{w}=1$ at $S_{l}=S_{l}{ }^{*}$. Physically, one must have $k_{r l}{ }^{w}$ $=1$ at $S_{l}=1$. A convenient means to achieve this is to use the drainage branch $k_{r l}{ }^{d}$ for both drainage and imbibition when $S_{l}>S_{l}^{*}$. To obtain a smooth connection between $k_{r l}{ }^{w}$ and $k_{r l}{ }^{d}$, a small cubic spline is introduced. The user specifies the width over which the spline extends. 


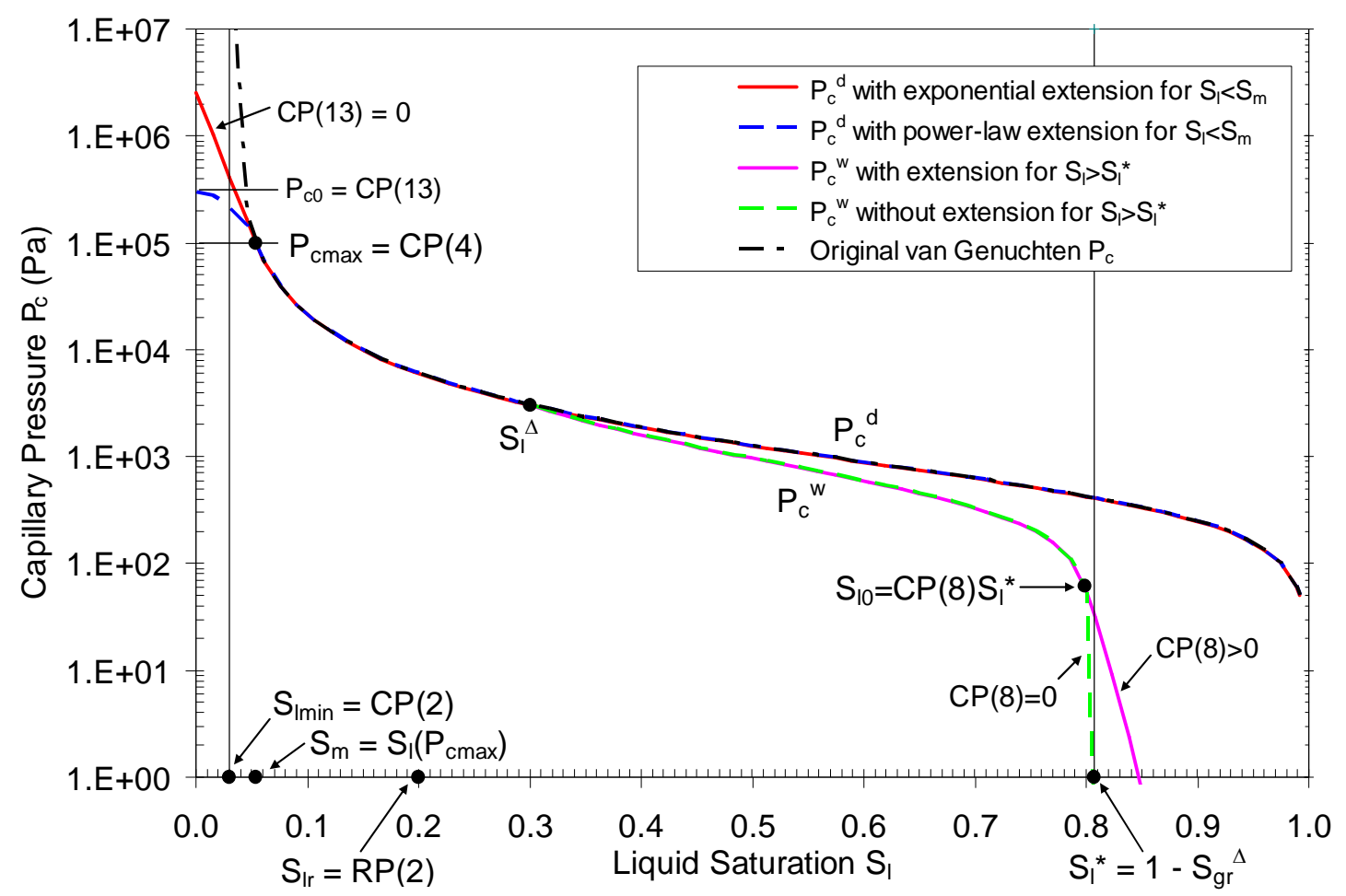

Figure 1. Features of the extended hysteretic capillary pressure function. $P_{c}{ }^{w}$ is shown for a typical value of $S_{l}^{\Delta}=0.3$. In practice, $S_{l}^{\Delta}$ can range from $S_{l r}$ to 1 , with $S_{l}^{*}$ increasing as $S_{l}^{\Delta}$ increases. 


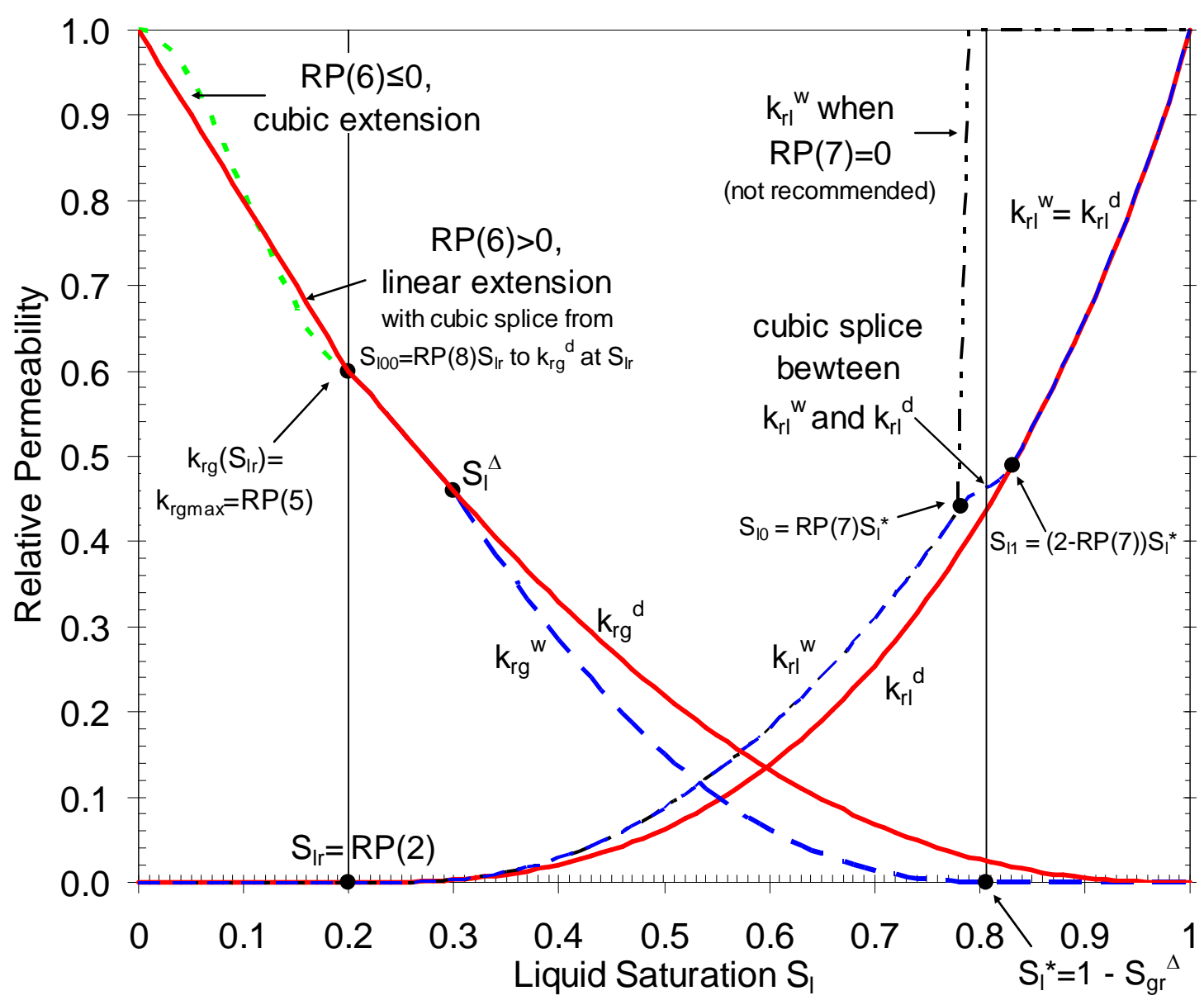

Figure 2. Features of the extended hysteretic relative permeability functions.

\section{Input Parameters}

\subsection{Characteristic Curves}

To accommodate the parameters required to specify hysteretic problems, two lines are read in for both relative permeability parameters and capillary pressure parameters. For non-hysteretic materials (IRP $\neq 12, \mathrm{ICP} \neq 12)$, the second line should not be present. The format for the first line is the usual (I5,5X,7E10.4) and the format for the second line is (8E10.4). 


\section{IRP = $12 \quad$ (must use with ICP = 12)}

$\mathrm{RP}(1)=m$; van Genuchten $m$ for liquid relative permeability (need not equal $\mathrm{CP}(1)$ or $\mathrm{CP}(6)) ; k_{r l}$ uses same $m$ for drainage and imbibition.

$\mathrm{RP}(2)=S_{l r}$; residual liquid saturation: $k_{r l}\left(S_{l r}\right)=0, k_{r g}\left(S_{l r}\right)=k_{r g \max }$. Must have $S_{l r}>S_{l m i n}$ in capillary pressure $(\mathrm{CP}(2)) . S_{l r}$ is minimum saturation for transition to imbibition branch. For $S_{l}<S_{l r}$, curve stays on primary drainage branch even if $S_{l}$ increases.

$\mathrm{RP}(3)=S_{\text {grmax }}$; maximum possible value of residual gas saturation $S_{g r}{ }^{\Delta}$. Note that the present version of the code requires that $S_{l r}+S_{g r \max }<1$, otherwise there will be saturations for which neither fluid phase is mobile, which the code cannot handle. Setting $S_{g r \max }=0$ effectively turns off hysteresis. As a special option, a constant, non-zero value of $S_{\mathrm{gr}}$ may be employed by setting CP(10)>1 and making RP(3) negative. The code will set $S_{g r}{ }^{\Delta}=-\mathrm{RP}(3)$ for all grid blocks at all times.

$\mathrm{RP}(4)=\gamma$; exponent in $k_{r g}$, typical values $0.33-0.50$. In Doughty (2007), $\gamma$ was hardwired at $1 / 3$.

$\mathrm{RP}(5)=k_{r g \max }=k_{r g}\left(S_{l r}\right)$; maximum value of $k_{r g}$ obtained using Equation (3). In Doughty (2007), $k_{\text {rgmax }}$ was hardwired at 1.

$\mathrm{RP}(6)=$ fitting parameter for $k_{r g}$ extension for $S_{l}<S_{l r}$ (only used when $k_{r g \max }<1$ ); determines type of function for extension and slope of $k_{r g}$ at $S_{l}=0$. $\leq 0 \quad$ use cubic spline for $0<S_{l}<S_{l r}$, with slope at $S_{l}=0$ of $\operatorname{RP}(6)$ $>0$ use linear segment for $0<S_{l}<\mathrm{RP}(8) S_{l r}$ and cubic spline for $\mathrm{RP}(8) S_{l r}<S_{l}<S_{l r}$, with slope at $S_{l}=0$ of $-\mathrm{RP}(6)$.

$\mathrm{RP}(7)=$ numerical factor used for $k_{r l}$ extension to $S_{l}>S_{l}{ }^{*} . \operatorname{RP}(7)$ is the fraction of $S_{l}^{*}$ at which $k_{r l}$ curve departs from the original van Genuchten function. Recommended range of values: $0.95-0.97$. For $\operatorname{RP}(7)=0, k_{r l}=1$ for $S_{l}>S_{l}^{*}$ (not recommended).

$\mathrm{RP}(8)=$ numerical factor used for linear $k_{r g}$ extension to $S_{l}<S_{l r}$ (only used when $k_{r g m a x}<$

1). $\mathrm{RP}(8)$ is the fraction of $S_{l r}$ at which the linear and cubic parts of the extensions are joined.

$\mathrm{RP}(9)=$ flag to turn off hysteresis for $k_{r l}$ (no effect on $P_{c}$ and $k_{r g}$; to turn off hysteresis entirely, set $S_{\text {grmax }}=0$ in $\operatorname{RP}(3)$ ).

$=0 \quad$ hysteresis is on for $k_{r l}$

$=1 \quad$ hysteresis is off for $k_{r l}$ (force $k_{r l}$ to stay on primary drainage branch $\left(k_{r l}{ }^{d}\right)$ at all times)

$\mathrm{RP}(10)=m_{\text {gas }}$; van Genuchten $m$ for gas relative permeability (need not equal $\mathrm{CP}(1)$ or $\mathrm{CP}(6)) ; k_{r g}$ uses same $m_{g a s}$ for drainage and imbibition. If zero or blank, use $\mathrm{RP}(1)$ so that $m_{\text {gas }}=m$. 


\section{ICP $=12$ (must use with IRP $=12$, uses parameters $\mathrm{RP}(2)$ and $\mathrm{RP}(3)$ )}

$\mathrm{CP}(1)=m^{d}$; van Genuchten $m$ for drainage branch $P_{c}^{d}\left(S_{l}\right)$.

$\mathrm{CP}(2)=S_{l \min }$; saturation at which original van Genuchten $P_{c}$ goes to infinity. Must have $S_{l \min }<S_{l r}$, where $S_{l r}$ is the relative permeability parameter RP(2).

$\mathrm{CP}(3)=P_{0}{ }^{d}$; capillary strength parameter for drainage branch $P_{c}{ }^{d}\left(S_{l}\right)(\mathrm{Pa})$.

$\mathrm{CP}(4)=P_{c \max }$; maximum capillary pressure $(\mathrm{Pa})$ obtained using original van Genuchten $P_{c}$ (Equation 1). Inverting Equation (1) for $P_{c \max }$ determines $S_{m}$, the transition point between the original van Genuchten function and an extension that stays finite as $S_{l}$ goes to zero. For functional form of extension, see description of $\mathrm{CP}(13)$ below.

$\mathrm{CP}(5)=$ scale factor for pressures for unit conversion (1 for pressure in $\mathrm{Pa}$ ).

$\mathrm{CP}(6)=m^{w}$; van Genuchten $m$ for imbibition branch $P_{c}{ }^{w}\left(S_{l}\right)$. Default value is $\mathrm{CP}(1)$ (recommended unless compelling reason otherwise).

$\mathrm{CP}(7)=P_{0}{ }^{w}$; capillary strength parameter for imbibition branch $P_{c}{ }^{w}\left(S_{l}\right)(\mathrm{Pa})$. Default value is $\mathrm{CP}(3)$ (recommended unless compelling reason otherwise).

$\mathrm{CP}(8)=$ parameter indicating whether to invoke non-zero $P_{c}$ extension for $S_{l}>S_{l}^{*}=1-$ $S_{g r}{ }^{\Delta}$

$=0 \quad$ no extension; $P_{c}=0$ for $S_{l}>S_{l}^{*}$

$>0 \quad$ power-law extension for $S_{l}{ }^{*}<S_{l}<1$, with $P_{c}=0$ when $S_{l}=1$. A non-zero $\mathrm{CP}(8)$ is the fraction of $S_{l}{ }^{*}$ at which the $P_{c}$ curve departs from the original van Genuchten function. Recommended range of values: 0.97-0.99.

$\mathrm{CP}(9)=$ flag indicating how to treat negative radicand, which can arise for $S_{l}>S_{l}^{\Delta 23}$ for second-order scanning drainage curves (ICURV $=3$ ), where $S_{l}^{\Delta 23}$ is the turningpoint saturation between first-order scanning imbibition $($ ICURV $=2$ ) and second-order scanning drainage. None of the options below have proved to be robust under all circumstances. If difficulties arise because $S_{l}>S_{l}^{\Delta 23}$ for ICURV $=3$, also consider using IEHYS $(3)>0$ or $\mathrm{CP}(10)<0$, which should minimize the occurrence of $S_{l}>S_{l}^{\Delta 23}$ for ICURV $=3$.

$=0 \quad$ radicand $=\max (0$,radicand $)$ regardless of $S_{l}$ value

$=1 \quad$ if $S_{l}>S_{l}^{\Delta 23}$, radicand takes value of radicand at $S_{l}=S_{l}^{\Delta 23}$

$=2 \quad$ if $S_{l}>S_{l}^{\Delta 23}$, use first-order scanning imbibition curve $($ ICURV $=2)$

$\mathrm{CP}(10)=$ threshold value of $|\Delta S|$ (absolute value of saturation change since previous time step) for enabling a branch switch (default is $1 \mathrm{E}-6$; set to any negative number 
to do a branch switch no matter how small $|\Delta S|$ is; set to a value greater than 1 to never do a branch switch). See also IEHYS(3).

$\mathrm{CP}(11)=$ threshold value of $S_{g r}{ }^{\Delta}$. If value of $S_{g r}{ }^{\Delta}$ calculated from $S_{l}{ }^{\Delta}$ (Equation (2)) is less than CP(11), use $S_{g r}{ }^{\Delta}=0$. Recommended value 0.01-0.03; default is 0.02 .

$\mathrm{CP}(12)=$ flag to turn off hysteresis for $P_{c}$ (no effect on $k_{r l}$ and $k_{r g}$; to turn off hysteresis entirely, set $S_{\text {grmax }}=0$ in $\left.\operatorname{RP}(3)\right)$.

$=0 \quad$ hysteresis is on for $P_{c}$

$=1 \quad$ hysteresis is off for $P_{c}$ (switch branches of $P_{c}$ as usual, but set $S_{g r}=0$ in $P_{c}$ calculation. Make sure other parameters of $P_{c}{ }^{d}$ and $P_{c}{ }^{w}$ are the same: $\mathrm{CP}(1)=\mathrm{CP}(6)$ and $\mathrm{CP}(3)=\mathrm{CP}(7))$

$\mathrm{CP}(13)=$ parameter to determine functional form of $P_{c}$ extension for $S_{l}\left\langle S_{l \min }\right.$ (i.e., $\left.P_{c}\right\rangle$ $\left.P_{c \max }\right)$

$=0 \quad$ exponential extension

$>0 \quad$ power-law extension with zero slope at $S_{l}=0$ and $P_{c}(0)=\mathrm{CP}(13)$.

Recommended value: 2 to 5 times $\mathrm{CP}(4)=P_{c \max }$. Should not be less than or equal to $\mathrm{CP}(4)$.

\section{Comparison of Hysteretic and Non-Hysteretic Characteristic Curves}

The non-hysteretic characteristic curves most comparable to the hysteretic curves described above are the van Genuchten (1980) curves (IRP = ICP =7). The drainage branches $P_{c}^{d}$ and $k_{r l}{ }^{d}$ of IRP $=\mathrm{ICP}=12$ are identical to $P_{c}$ and $k_{r l}$ of IRP $=\mathrm{ICP}=7$ (but the manner of defining input parameters differs, so the ROCKS or RPCAP blocks need to be modified). However, the functional form of $k_{r g}$ differs for IRP $=7$ and IRP $=12$, so even for problems involving only drainage, hysteretic and non-hysteretic cases will not produce identical results.

There are several ways to effectively turn off hysteresis in a material with IRP $=$ ICP $=12$, which may be useful for evaluating the impact of hysteresis for a given problem.

One can set $S_{\text {grmax }}=\mathrm{RP}(3)=0, \mathrm{CP}(1)=\mathrm{CP}(6)$, and $\mathrm{CP}(3)=\mathrm{CP}(7)$. The code will identify turning points and switch from branches as usual, but all the branches will 
overlie the primary drainage branch. Alternatively, one can set $\mathrm{CP}(10)$, the threshold value of $|\Delta S|$ for switching branches, to a value greater than 1.0. Then each grid block will remain on whatever branch it begins on, and its value of $S_{g r}{ }^{\Delta}$ will not change (must have IEHYS(3) $=0$, see below). Finally, a special option exists to enable a constant, nonzero value of $S_{g r}{ }^{\Delta}$ to be applied to all grid blocks for all times. This is invoked by setting $\mathrm{CP}(10)>1.0, \operatorname{IEHYS}(3)=0$, and $\mathrm{RP}(3)=-S_{g r}{ }^{\Delta}$.

\subsection{HYSTE Block Parameters}

The HYSTE block is used to provide some numerical controls on the hysteretic characteristic curves. It is not needed if the default values of all its parameters are to be used. The format is (3I5).

$\operatorname{IEHYS}(1)=$ flag to print information about hysteretic characteristic curves $=0 \quad$ no additional print out

$\geq 1$ print a one-line message to the output file every time a capillary-pressure branch switch occurs (recommended)

$\geq 2$ print a one-line message to the iTOUGH2 message (.msg) file every time the third-order scanning imbibition curve crosses either the first-order scanning imbibition curve or the second-order scanning drainage curve

$\operatorname{IEHYS}(2)=$ flag indicating when to apply capillary-pressure branch switching

$=0 \quad$ after convergence of time step (recommended)

$>0$ after each Newton-Raphson iteration

IEHYS(3) = run parameter for sub-threshold saturation change

$=0 \quad$ no branch switch unless $|\Delta S|>\mathrm{CP}(10)$

$>0$ allow branch switch after run of IEHYS(3) consecutive time steps for which all $|\Delta S|<\mathrm{CP}(10)$ and all $\Delta S$ are the same sign. Recommended value 5-10. This option may be useful if the time step is cut to a small value due to convergence problems, making saturation changes very small.

\subsection{MOP Parameter}

$\operatorname{MOP}(13)=$ Flag to control how INCON block is read 
$=0$ or 1 read normal INCON block with two lines per element

$=2$ read hysteretic INCON block with seven lines per element. The additional five lines contain history information for elements with ICP $=$ IRP $=12$. For non-hysteretic materials, no history information is required; the additional five lines contain zeros and these entries are not used.

Note that if $\mathrm{MOP}(13)=0$ or 1 or if an element does not appear in the INCON block (or if no INCON block is present), then no history information is available. For elements with ICP $=$ IRP $=12$, the only initial conditions that can be specified without history information are for single-phase liquid $\left(P_{c}=0\right)$, or for the primary drainage branch of the capillary pressure curve (two-phase liquid-gas or single-phase gas). Initial conditions on imbibition branches or the scanning drainage branch require that $\mathrm{MOP}(13)=2$ so that all relevant history information will be available. The only practical way to obtain this history information is to use the SAVE file from a prior simulation as the INCON block.

\section{Output}

\subsection{Every Time Step}

The message printed out each iteration when $\mathrm{MOP}(1)>0$ includes the variable KTMP0, the temporary value of the variable ICURV, which identifies which branch of the capillary pressure curve is being followed: 0 - single-phase liquid, 1 - primary drainage, 2 - first-order scanning imbibition, 3 - second-order scanning drainage, 4 third-order scanning imbibition.

If IEHYS $(1)>0$, a one-line message is printed each time an element undergoes a branch switch, including the element name, the ICURV value for the old and new branches, and the turning-point saturation $\left(S_{l}^{\Delta 12}, S_{l}^{\Delta 23}\right.$, or $\left.S_{l}^{\Delta 34}\right)$. This information can be 
very useful for analyzing simulations that take small time steps due to slow convergence. When time step is small, many successive small (sub-threshold) saturation changes that are individually too small to trigger a branch switch may occur. This can lead to a saturation on the "wrong side" of the turning-point saturation, which can slow convergence. A typical manifestation of this situation is the maximum residual repeatedly occurring at an element that has ICURV=3. Search backward through the output file to find the most recent branch switch for that element (look for the string "element-name old curve") and compare the current $S_{l}$ to $S_{l}^{\Delta 23}$. If $S_{l}>S_{l}^{\Delta 23}$, it may be worthwhile to stop the code, modify option $\mathrm{CP}(9), \mathrm{CP}(10)$, or IEHYS(3), and restart the code using the SAVE file as the new INCON file.

\subsection{Main Output}

Columns have been added to show ICURV, the branch of the capillary pressure curve being followed, and SOR, the history-dependent value of residual gas saturation $\mathrm{S}_{\mathrm{gr}}{ }^{\Delta}$.

\subsection{SAVE File}

The SAVE file must contain all the information required to do a restart, thus whenever there is at least one hysteretic material $(\mathrm{IRP}=\mathrm{ICP}=12)$ in a problem, the save file contains five lines for each element with information on capillary-pressure branch, turning points, etc., in addition to the usual two lines containing element name, porosity, and state variables. For non-hysteretic materials (IRP, ICP $\neq 12$ ), the entries in the 
additional five lines are all zero and are not used. These unused lines are included so that the SAVE file may be used as an INCON file for a subsequent restart.

\subsection{Additional Files}

File.hys (unit 82) If an element ELST is specified in the PARAM block, and that element has IRP $=\mathrm{ICP}=12$, then this file contains the values of time, $S_{l}, P_{c}, \mathrm{ICURV}, k_{r g}$, and $k_{r l}$ for that element every time step. The file is formatted for Tecplot, but the user needs to edit the file to add the number of entries (after " $i=$ " on line 3 ), and optionally add title information within the pairs of quotes on lines 1 and 3.

iTOUGH2file.msg If IEHYS $(1) \geq 2$, a message is printed every time the thirdorder scanning imbibition curve (ICURV $=4)$ crosses either the first-order scanning imbibition curve (ICURV = 2) or the second-order scanning drainage curve (ICURV = 3). Warning: this file can get very big, and probably is only useful for debugging purposes.

\section{Sample Problems}

\subsection{Problem 1: $\mathrm{CO}_{2}$ Injection with Dry Out and Subsequent Dissolution}

A simple one-dimensional problem showing $\mathrm{CO}_{2}$ injection into a tilted saline aquifer is presented to demonstrate the hysteretic version of iTOUGH2 with the EOS17 $(\mathrm{ECO} 2 \mathrm{~N})$ module, in particular to test the extensions beyond which brine and $\mathrm{CO}_{2}$ are immobile. Two cases consider different rates and durations of $\mathrm{CO}_{2}$ injection. In the first case, a relatively small amount of $\mathrm{CO}_{2}$ is injected to illustrate a range of $S_{g r}{ }^{\Delta}$ values. In the second case, $\mathrm{CO}_{2}$ injection is large enough for elements near the injection location to 
dry out. In both cases, the simulations continue after $\mathrm{CO}_{2}$ injection ceases. Hysteretic $(\mathrm{IRP}=\mathrm{ICP}=12)$ and non-hysteretic $(\mathrm{IRP}=\mathrm{ICP}=7)$ cases are shown to enable comparison. Table 1 summarizes numerical performance of the cases run. See the input files for detailed specification of material properties, including characteristic curves. Parameters are believed reasonable for high-permeability saline formations, and are similar to parameters used to model the Frio brine pilot (Doughty et al., 2008).

Table 1. Job name and CPU time required for 100-year simulations of Problem 1, using a Dell Latitude D800 with $2 \mathrm{GHz}$ clock speed and 2GB RAM.

\begin{tabular}{|l|l|l|}
\hline Amount of $\mathrm{CO}_{2}$ injected & Hysteretic & Non-hysteretic \\
\hline $1 \mathrm{~kg} / \mathrm{s} \mathrm{CO}_{2}$ for 1 day & $\mathrm{j} 203 \mathrm{t}-356 \mathrm{steps}, 18 \mathrm{sec}$ & $\mathrm{d} 2 \mathrm{o} 3 \mathrm{t}-304 \mathrm{steps}, 13 \mathrm{sec}$ \\
\hline $5 \mathrm{~kg} / \mathrm{s} \mathrm{CO} 2$ for 5 days & h2o3t $-398 \mathrm{steps}, 21 \mathrm{sec}$ & $\mathrm{c} 2 \mathrm{o} 3 \mathrm{t}-392 \mathrm{steps}, 17 \mathrm{sec}$ \\
\hline
\end{tabular}

The model is one-dimensional and consists of 100 elements, each $2 \mathrm{~m}$ long, tilted at an angle of $10^{\circ}$ from the horizontal. $\mathrm{CO}_{2}$ is injected at a constant rate near the middle of the model. Prior to $\mathrm{CO}_{2}$ injection, a gravity-equilibration simulation is done for a brine with $P=200$ bars, $T=75^{\circ} \mathrm{C}$, and a salt content of $\mathrm{X}_{\mathrm{NaCl}}=0.1$. Then, the lowermost and uppermost elements are made inactive to hold pressure fixed at the resulting values. Next, the amount of salt in every element is decreased to $\mathrm{X}_{\mathrm{NaCl}}=0.03$, which results in lower density and a moderate steady upward brine flow through the model. These are the initial conditions for the $\mathrm{CO}_{2}$ injection. The upward brine flow was chosen to illustrate the dissolution of immobile $\mathrm{CO}_{2}$.

\section{Results}

Figures 3 and 4 show the hysteretic characteristic curves for the injection element for cases j203t and h203t. Figures 5 and 6 show profiles of gas saturation for a series of times, comparing hysteretic and non-hysteretic cases. In all cases, by the end of the 100- 
year simulation period, all $\mathrm{CO}_{2}$ has been purged from the system. For the non-hysteretic cases, which have $S_{g r}=0$, much of the $\mathrm{CO}_{2}$ flows out of the model as a free phase. For the hysteretic cases, which have $S_{g r \max }=0.2$, a significant fraction of the injected $\mathrm{CO}_{2}$ becomes immobilized, but it gradually dissolves in the upward-flowing brine that is moving past the trapped $\mathrm{CO}_{2}$ plume, and eventually flows out of the model in dissolved form.

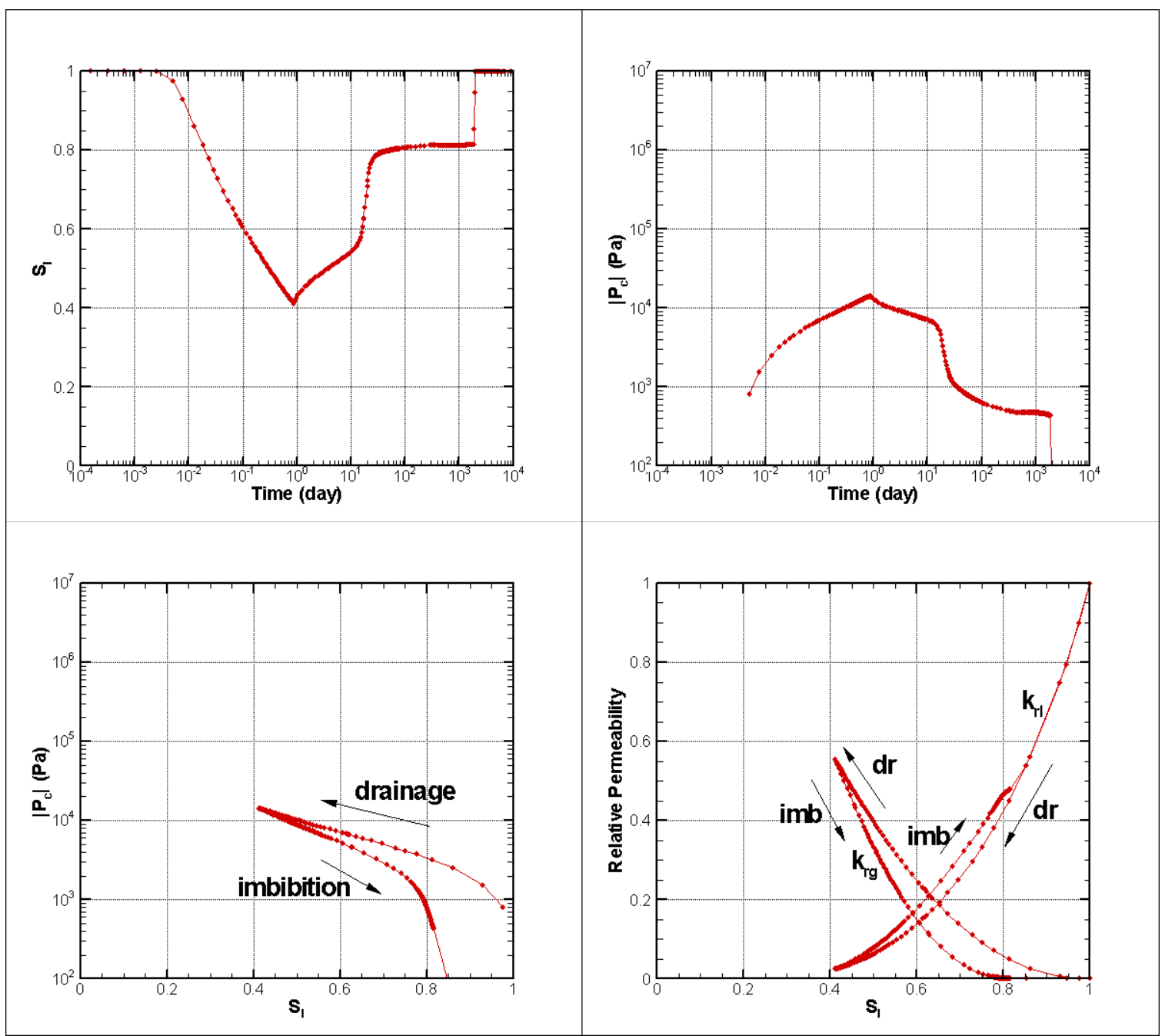

Figure 3. Characteristic curve branches followed for the injection element for Case j203t, in which a small amount of $\mathrm{CO}_{2}$ is injected for 1 day, followed by 100 years of redistribution. 


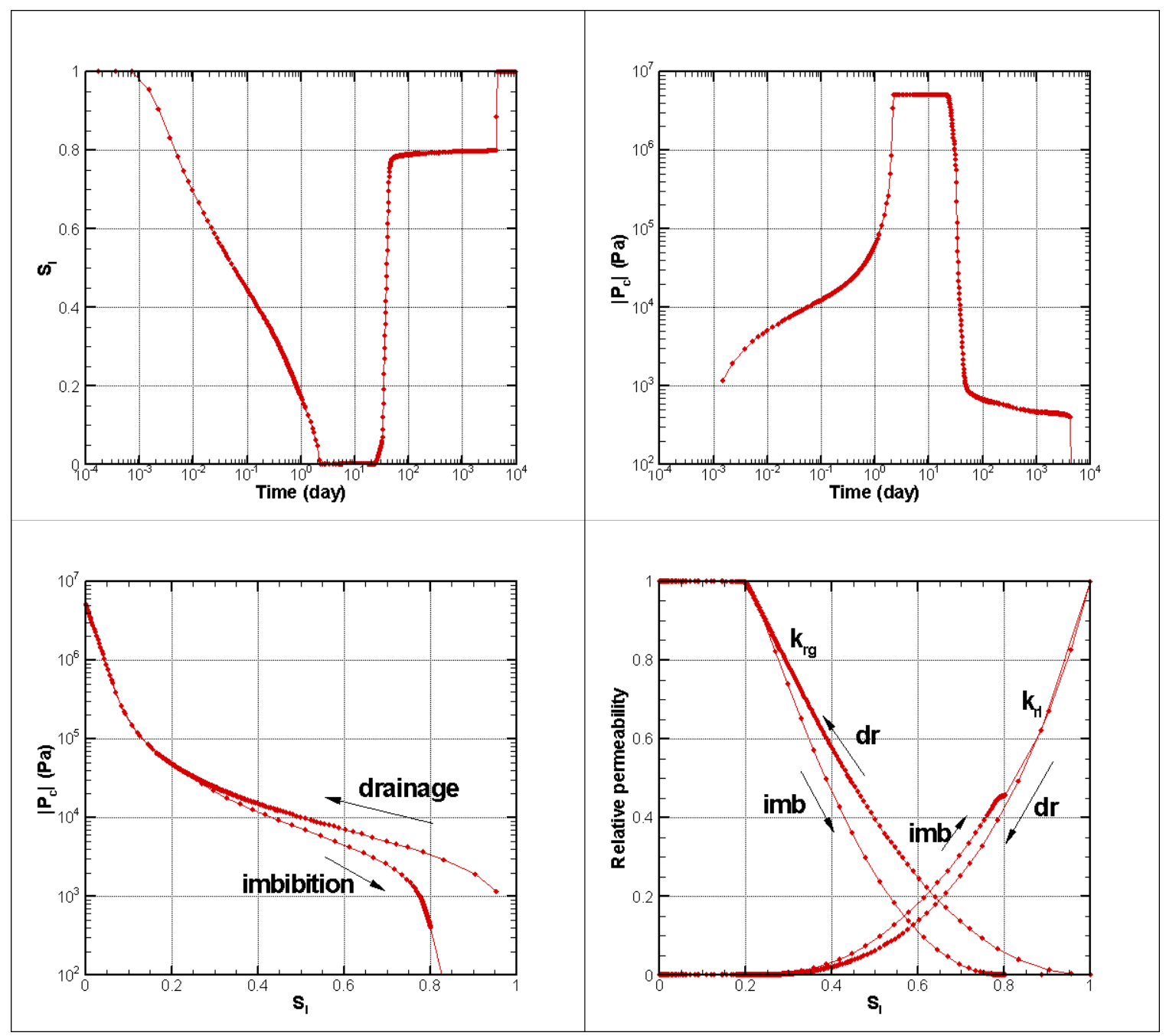

Figure 4. Characteristic curve branches followed for the injection element for Case h203t, in which a large amount of $\mathrm{CO}_{2}$ is injected for 10 days, followed by 100 years of redistribution. 

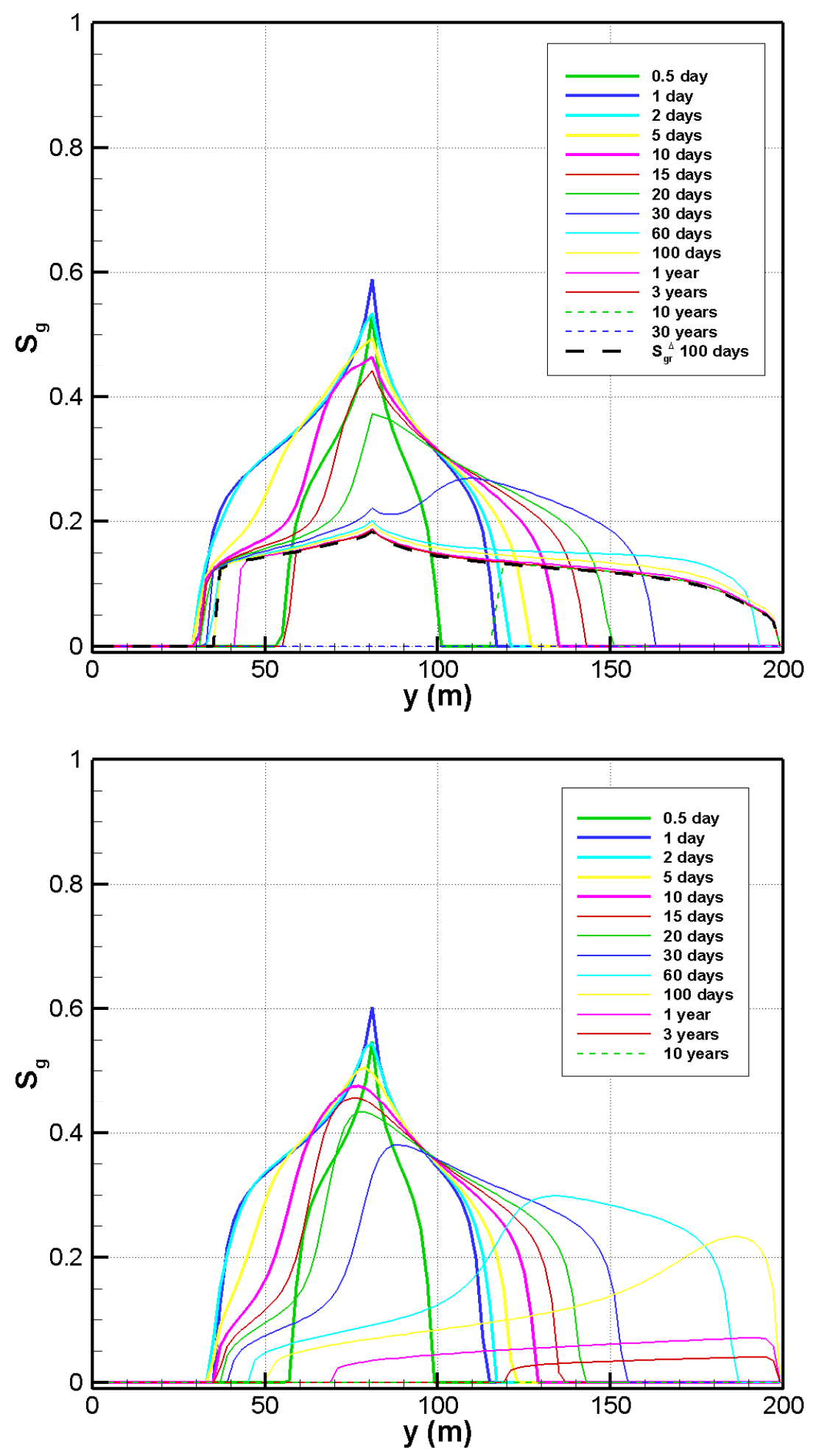

Figure 5. Profiles of $S_{g}$ versus distance along the model for various times for cases with a small amount of $\mathrm{CO}_{2}$ injected: j203t (with hysteresis, top) and d203t (no hysteresis, bottom). The injection element is located at $y=81 \mathrm{~m}$. For the hysteretic case, $S_{g r}{ }^{\Delta}$ at 100 days is also shown. 

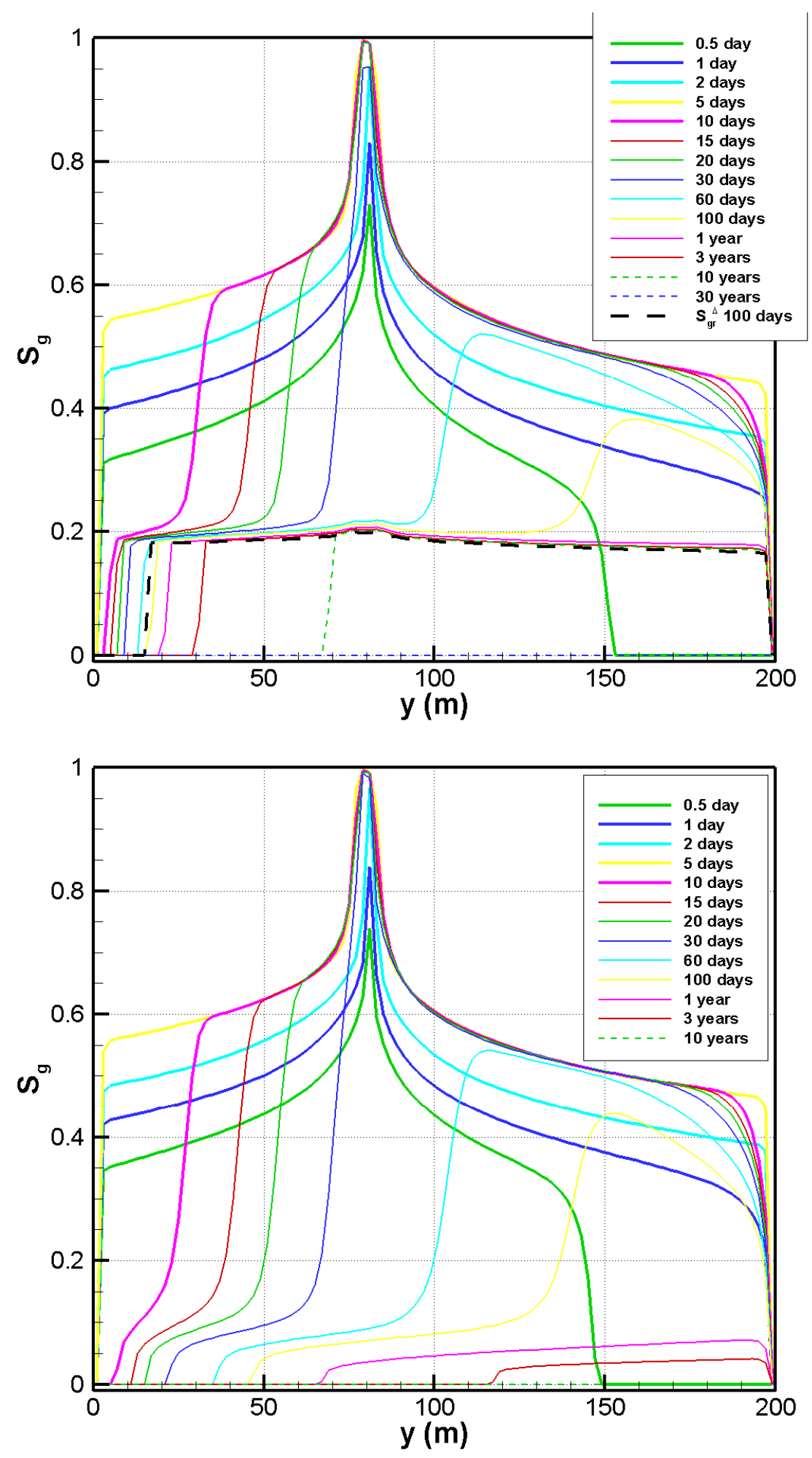

Figure 6. Profiles of $S_{g}$ versus distance along the model for various times for cases with a large amount of $\mathrm{CO}_{2}$ injected: h203t (with hysteresis, top) and c203t (no hysteresis, bottom). The injection element is located at $y=81 \mathrm{~m}$. For the hysteretic case, $S_{g r}{ }^{\Delta}$ at 100 days is also shown. 


\subsection{Problem 2: Sequence of Repeated $\mathrm{CO}_{2}$ and $\mathrm{H}_{2} \mathrm{O}$ Injection Periods}

Problem 2 (developed by Michele Carpita and Alfredo Battistelli of Snamprogetti, Italy) considers a series of successive $\mathrm{CO}_{2}$ and water injections inside a $240 \mathrm{~m}$ long, onedimensional, horizontal grid without salt and under isothermal conditions. The whole simulation lasts just 60 days, and there is no resting time between any two subsequent injections. The whole grid is initialized at $P=100$ bar and $T=50{ }^{\circ} \mathrm{C}$ conditions. A boundary element with a fixed pressure of $P=100$ bar is located at $x=0$ in order to induce all the injected fluids to move toward it.

The injection sequence was created in order to test all the branches of the hysteretic curves and the branch-switching processes, without the additional complications of Problem 1 (e.g., gravity flow of both brine and $\mathrm{CO}_{2}, \mathrm{CO}_{2}$ solubility dependence on salt content). Each injection element is located upgradient with respect to the previous injection element, to avoid spurious effects in the elements far from the fixed boundary.

The injection sequence consists of five steps, detailed as follows:

1) - A first $\mathrm{CO}_{2}$ injection ( $2 \mathrm{~kg} / \mathrm{s}$ for 2 days) in element $\mathrm{A} 1146(x=178 \mathrm{~m})$ in order to place the nearby elements along the primary drainage curve $($ ICURV $=1)$.

2) - A first $\mathrm{H}_{2} \mathrm{O}$ injection $(0.01 \mathrm{~kg} / \mathrm{s}$ for 23 days) in element $\mathrm{A} 1149(x=190 \mathrm{~m})$ to put some elements on the first-order imbibition curve $($ ICURV $=2)$.

3) - Another $\mathrm{CO}_{2}$ injection $(0.5 \mathrm{~kg} / \mathrm{s}$ for 5 days $)$ in element $\mathrm{A} 1152(x=202 \mathrm{~m})$ to activate the second-order drainage curve (ICURV $=3$ ). 
4) - A second $\mathrm{H}_{2} \mathrm{O}$ injection $(0.5 \mathrm{~kg} / \mathrm{s}$ for 20 days $)$ in element $\mathrm{A} 1155(x=214 \mathrm{~m})$ to activate the third-order imbibition curve (ICURV $=4$ ). The $\mathrm{H}_{2} \mathrm{O}$ injection is prolonged so as to let the water saturation increase above the domain of the second-order drainage

curve (i.e., to $S_{l}>S_{l}^{\Delta 23}$ ), to check if there is a correct branch switch to the first-order imbibition curve (ICURV $=2)$.

5) - A final $\mathrm{CO}_{2}$ injection $(1 \mathrm{~kg} / \mathrm{s}$ for 10 days $)$ in element $\mathrm{A} 1158(x=226 \mathrm{~m})$ to see what happens after the third-order imbibition.

\section{Results}

Figure 7 shows a series of snapshots of the saturation distribution in the model. The overall flow of fluid toward the constant-pressure boundary at $x=0$ is evident. Element A1140, which is located at $x=154 \mathrm{~m}$, just downstream of all the injection elements, is chosen as the primary location at which to examine the saturation and capillary pressure variation more closely, as shown in Figure 8. 


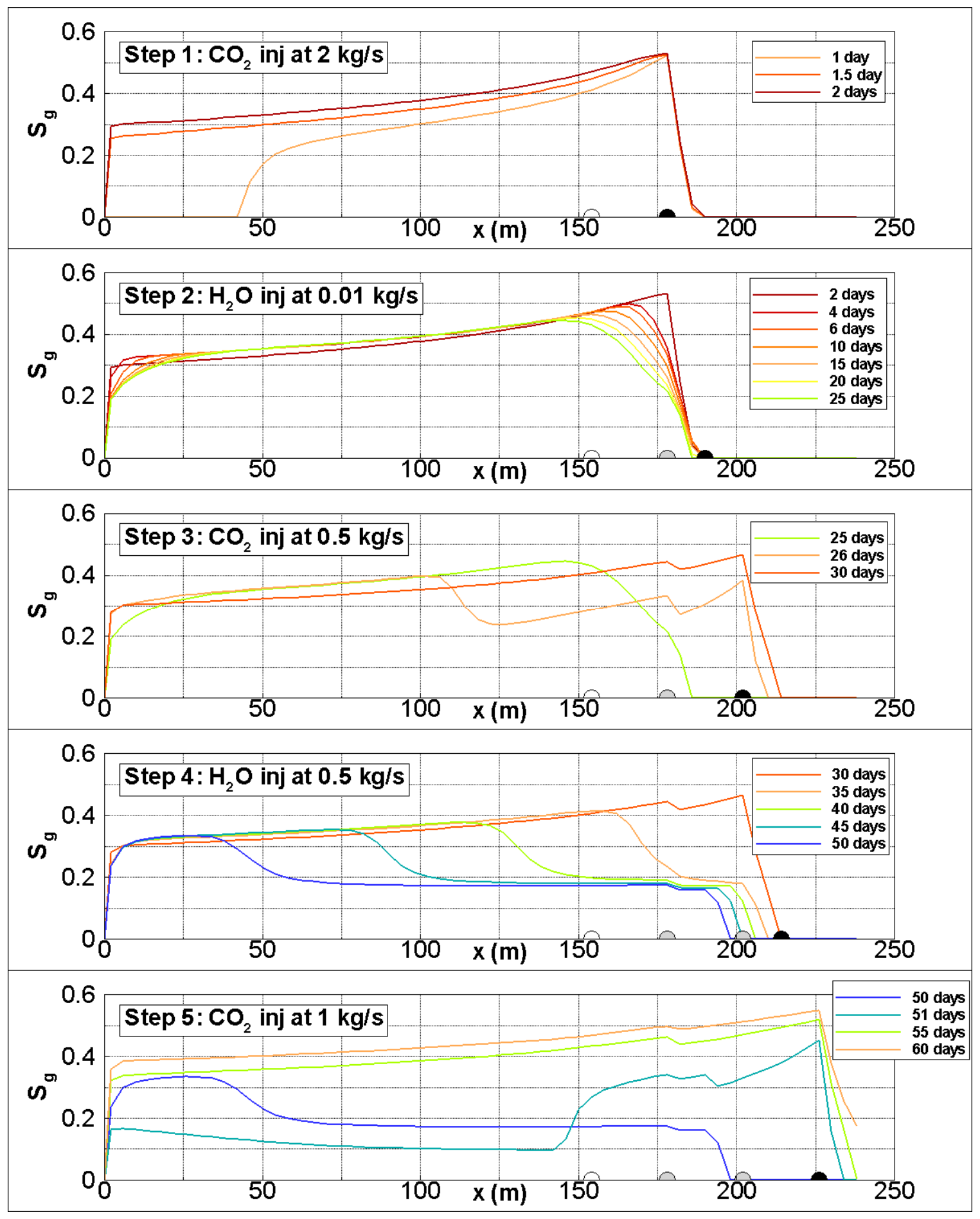

Figure 7. Saturation distributions in the model at various times during the five-step injection sequence. Each injection location is identified with a filled black or grey dot. The location of element A1140 is shown as an open dot. 


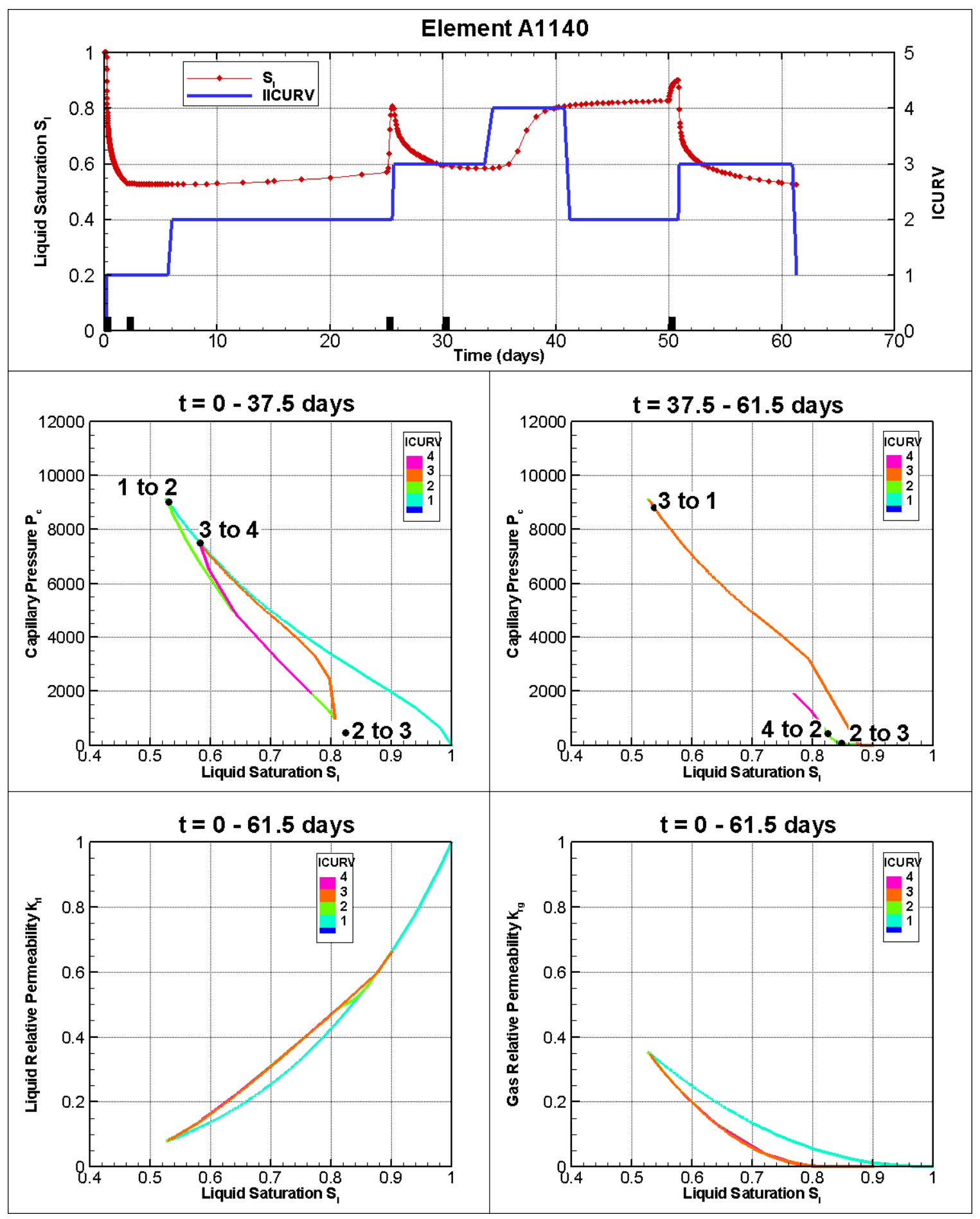

Figure 8. Top: time-variation of liquid saturation and capillary-pressure-curve branch (ICURV) for element A1140. The start of each injection step is marked by a black bar. Middle: capillary pressure versus liquid saturation at element A1140. The plot is divided into two parts, based on time, to enable the different branches of the capillary pressure curve to be seen more easily. The branch switches are marked by black dots, with ICURV for each switch labelled. Bottom: relative permeability versus liquid saturation at element A1140. Branches 2, 3, and 4 all overlie one another. 


\section{Acknowledgments}

Review of this document by Stefan Finsterle of Berkeley Lab is gratefully appreciated. Special thanks go to Michele Carpita and Alfredo Battistelli of Snamprogetti, who carefully examined the code and provided much valuable input for its improvement. This work was supported by the ZERT (Zero Emissions Research and Technology) and GEOSEQ programs, through the Assistant Secretary for Fossil Energy, Office of Coal and Power Systems through the National Energy Technology Laboratory, and by Lawrence Berkeley National Laboratory under Department of Energy Contract No. DE-AC02$05 \mathrm{CH} 11231$.

\section{References}

Doughty C., Modeling geologic storage of carbon dioxide: comparison of non-hysteretic and hysteretic characteristic curves. Energy Conversion and Management 48(6), 17681781, 2007.

Doughty C, Freifeld BM, Trautz RC. Site characterization for $\mathrm{CO}_{2}$ geologic storage and vice versa: The Frio brine pilot, Texas, USA as a case study. Environmental Geology 54(8), 1635-1656, DOI:10.1007/S00254-007-0942-0, 2008.

Finsterle S. iTOUGH2 user's guide. Rep. LBNL-40040, Lawrence Berkeley National Laboratory, Berkeley, CA, 1999a.

Finsterle S. iTOUGH2 sample problems. Rep. LBNL-40041, Lawrence Berkeley National Laboratory, Berkeley, CA, 1999b.

Finsterle S. iTOUGH2 command reference. Rep. LBNL-40041, Lawrence Berkeley National Laboratory, Berkeley, CA, 1999c.

Finsterle S, Sonnenborg TO, Faybishenko B. Inverse modeling of a multistep outflow experiment for determining hysteretic hydraulic properties. In Pruess K, editor. Proceedings of the TOUGH workshop '98, Rep. LBNL-41995, Lawrence Berkeley National Laboratory, Berkeley, CA, 1998, p. 250-256.

Land CS, Calculation of imbibition relative permeability for two- and three-phase flow from rock properties (SPE 1942), SPE Journal 8(2), 149-156, 1968.

Lenhard RJ, Parker JC. A model for hysteretic constitutive relations governing multiphase flow, 2. Permeability-saturation relations. Water Resources Research 23(12), 2197-205, 1987 
Niemi A, Bodvarsson GS. Preliminary capillary hysteresis simulations in fractured rocks, Yucca Mountain, Nevada. Journal of Contaminant Hydrology 3, 277-91, 1988.

Parker JC, Lenhard RJ. A model for hysteretic constitutive relations governing multiphase flow, 1. Saturation-pressure relations. Water Resources Research 23(12), 2187-96, 1987.

Pruess K. ECO2N: A TOUGH2 Fluid Property Module for Mixtures of Water, NaCl, and $\mathrm{CO}_{2}$. Rep. LBNL-57952, Lawrence Berkeley National Laboratory, Berkeley, CA, 2005.

Pruess K, Oldenburg C, Moridis G. TOUGH2 user's guide, version 2.0. Rep. LBNL43134, Lawrence Berkeley National Laboratory, Berkeley, CA, 1999.

van Genuchten MTh. A closed-form equation for predicting the hydraulic conductivity of unsaturated soils. Soil Science Society of America Journal 44(5), 892-898, 1980. 